\title{
Mechanical punctate pain threshold is associated with headache frequency and phase in patients with migraine
}

\author{
Li-Ling Hope Pan' ${ }^{D}$, Yen-Feng Wang ${ }^{1,2,3}$, Kuan-Lin Lai ${ }^{1,2,3,4}{ }_{\mathbb{D}}$, \\ Wei-Ta Chen ${ }^{1,2,3}$, Shih-Pin Chen ${ }^{1,2,3,4,5}$ (D), Yu-Hsiang Ling ${ }^{2}$ (D), \\ Li-Wei Chou ${ }^{6}$, Rolf-Detlef Treede ${ }^{7}$ and Shuu-Jiun Wang ${ }^{1,2,3}$ (D)
}

\begin{abstract}
Objective: Previous studies regarding the quantitative sensory testing are inconsistent in migraine. We hypothesized that the quantitative sensory testing results were influenced by headache frequency or migraine phase.

Methods: This study recruited chronic and episodic migraine patients as well as healthy controls. Participants underwent quantitative sensory testing, including heat, cold, and mechanical punctate pain thresholds at the supraorbital area (VI dermatome) and the forearm ( $\mathrm{TI}$ dermatome). Prospective headache diaries were used for headache frequency and migraine phase when quantitative sensory testing was performed.

Results: Twenty-eight chronic migraine, 64 episodic migraine and 32 healthy controls completed the study. Significant higher mechanical punctate pain thresholds were found in episodic migraine but not chronic migraine when compared with healthy controls. The mechanical punctate pain thresholds decreased as headache frequency increased then nadired. In episodic migraine, mechanical punctate pain thresholds were highest $(p<0.05)$ in those in the interictal phase and declined when approaching the ictal phase in both VI and TI dermatomes. Linear regression analyses showed that in those with episodic migraine, headache frequency and phase were independently associated with mechanical punctate pain thresholds and accounted for $29.7 \%$ and $38.9 \%$ of the variance in $\mathrm{VI}(p=0.003)$ and $\mathrm{TI}(p<0.00 \mathrm{I})$ respectively. Of note, unlike mechanical punctate pain thresholds, our study did not demonstrate similar findings for heat pain thresholds and cold pain thresholds in migraine.

Conclusion: Our study provides new insights into the dynamic changes of quantitative sensory testing, especially mechanical punctate pain thresholds in patients with migraine. Mechanical punctate pain thresholds vary depending on headache frequency and migraine phase, providing an explanation for the inconsistency across studies.
\end{abstract}

\section{Keywords}

Pain sensitivity, quantitative sensory testing, mechanical punctate pain threshold

Date received: 23 February 2020; revised: I April 2020; accepted: 10 April 2020

\section{Introduction}

Migraine is one of the most common and disabling disorders (1). Altered cortical activity (2-4) and processing of sensory stimuli (5) have been reported in patients with migraine. Quantitative sensory

\footnotetext{
'Brain Research Center, National Yang-Ming University, Taipei, Taiwan ${ }^{2}$ Department of Neurology, Neurological Institute, Taipei Veterans General Hospital, Taipei, Taiwan

${ }^{3}$ Faculty of Medicine, School of Medicine, National Yang-Ming University, Taipei, Taiwan
}

\footnotetext{
${ }^{4}$ Institute of Clinical Medicine, National Yang-Ming University, Taipei, Taiwan

${ }^{5}$ Department of Medical Research, Taipei Veterans General Hospital, Taipei, Taiwan

${ }^{6}$ Department of Physical Therapy and Assistive Technology, National Yang-Ming University, Taipei, Taiwan

${ }^{7}$ Chair of Neurophysiology, Mannheim Center for Translational Neurosciences, Medical Faculty Mannheim, Heidelberg University, Germany
}

\section{Corresponding author:}

Shuu-Jiun Wang, Department of Neurology, Neurological Institute, Taipei Veteran General Hospital, No. 20I, Sec. 2, Shipai Rd., Beitou Dist., Taipei, Taiwan II2I7.

Email: sjwang@vghtpe.gov.tw 
testing (QST), which measures cutaneous sensory sensitivity changes and processing of sensory stimuli, have long been studied in patients with migraine (5-7). However, results are inconsistent. Some studies report hypersensitized cutaneous pain threshold, including heat-induced pain (8-12), cold-induced pain $(8,13)$, and pressure pain $(14,15)$; however, several other studies (10,16-19) suggest patients with migraine and controls have similar thresholds. Moreover, changes in punctate pain thresholds haven't been well addressed in this patient group compared with other stimuli.

The reasons for QST discrepancies in migraine patients are unknown. Few reports have studied the relationship between pain thresholds and migraine attacks. Because the characteristics of migraine are dynamic, sensitivity thresholds may change throughout the migraine phases cycle (e.g. interictal, preictal, ictal, and postictal phases). Many previous studies recruited migraine patients when they were headache free, but in some studies the migraine phase was not well classified. In addition, by influencing the cortical excitability, headache frequency may alter QST results. Two recent studies reported lower heat pain threshold (HPT) in migraine patients with higher headache frequency (20) or shorter interval to the next headache attack (9).

This study sought to investigate how headache frequency and migraine phase are related to pain sensitivity using heat-, cold-, and mechanical punctate-induced pain stimuli in migraine patients with varying headache frequencies and migraine phases. We hypothesized that pain sensitivity would be dynamic in patients with migraine regarding the headache attacks.

\section{Methods}

\section{Participants}

We recruited migraine patients from 2013 to 2018, who were diagnosed by board-certified neurologists at the Headache Clinic of Taipei Veterans General Hospital according to the International Classification of Headache Disorders (ICHD-3 beta or ICHD-3) $(21,22)$. Patients' demographics and clinical profiles were obtained from an intake form and verified faceto-face by neurologists. All migraine patients also completed the Migraine Disability Scale (MIDAS) to evaluate migraine-related disability in the prior 3 months and the Hospital Anxiety and Depression Scale (HADS) for anxious and depressive state in the prior week. In addition, sex- and age-matched healthy controls (HCs) without any history of migraine or other primary headaches were recruited. Infrequent tension-type headache ( $<1$ headache day/month) was, however, allowed. Of note, we only recruited patients whose major headache is migraine. Apart from the migraine diagnosis in the patient groups, both patients and HCs were normal in physical and neurological examinations and did not have any systemic diseases, major psychiatric disorders or conditions incompatible with the study.

None of the recruited migraine patients were taking migraine preventive agents; however, acute abortive medications were allowed but not on the day of QST. Patients were asked to document headache diaries for at least 4 weeks at baseline (i.e. prior to QST) and maintain the diaries for at least 2 weeks after QST to determine the migraine phase of the testing day (see below for definitions). The study protocol was approved by the Institutional Review Board at the Taipei Veterans General Hospital. All participants provided informed consent prior to participation. The study procedures followed the Declaration of Helsinki. Data from this study are available from the corresponding author upon request.

\section{Quantitative sensory testing}

The subjects were scheduled to undergo QST and were re-scheduled if abortive treatment was used within 3 days of testing. Participants laid comfortably in the supine position during the assessment. Assessment target areas included the left supraorbital (i.e. the first branch of the trigeminal nerve dermatome, V1) and proximal medio-ventral forearm (i.e. the first thoracic nerve dermatome, T1). There were breaks between each stimulus. All sensory stimuli types were delivered five times; the two extreme values were excluded, and the average values were calculated from the other three recordings for further analyses. The order of stimulation sites was randomized, while the order of stimulation types was fixed to reduce the intra-individual variance.

Heat and cold pain thresholds. Heat pain threshold (HPT) and cold pain threshold (CPT) were determined using the Medoc TSA-II NeuroSensory Analyzer (Medoc Ltd., Israel) with a $30 \mathrm{~mm} \times 30 \mathrm{~mm}$ Thermode placed on the testing area skin and secured with Velcro straps. To determine the HPT, five heat stimuli, starting at $32 \pm 0.5^{\circ} \mathrm{C}$ with a cut-off temperature of $50^{\circ} \mathrm{C}$, were given at the increasing rate of $1^{\circ} \mathrm{C}$ per second; and to determine CPT, five cold stimuli, starting at $32 \pm 0.5^{\circ} \mathrm{C}$ with a cut-off temperature at $0^{\circ} \mathrm{C}$, were given at the decreasing rate of $1^{\circ} \mathrm{C}$ per second (23).

Mechanical punctate pain threshold. Mechanical punctate stimuli were given with the standard rigid electronic 
von Frey filament (ITTC 2392, IITC Life Science Inc., USA). The mechanical punctate pain threshold (MPPT) was defined as the lowest intensity perceived as painful for participants. Participants were instructed to inform the examiner immediately upon painful sensation. Thresholds were determined with five series of ascending stimulus intensity, applied at $25 \mathrm{~g} / \mathrm{s}$ (24).

\section{Headache frequency and migraine phases}

Prospective paper headache diaries were used to determine headache frequency (i.e. number of headache days per 4 weeks, designated as monthly headache days, MHD) (25) before QST. Participants who completed less than $75 \%$ of the baseline headache diary were excluded from the analyses. Participants' migraine phases of the QST day were determined based on their headache diaries: a) preictal phase defined as within 72 hours of the next headache, b) postictal phase defined as within 24 hours following the headache, c) ictal phase defined as during headache attacks, and d) interictal phase.

\section{Statistical analysis}

All statistical analyses were performed in SPSS version 22.0 (IBM, Armonk, NY, USA). Correlation and linear regression analyses assessed headache profiles and pain thresholds. Continuous variables were compared between groups, namely episodic migraine (EM), chronic migraine (CM) and $\mathrm{HC}$ groups, with t-tests or one-way analysis of variance (ANOVA) tests with posthoc least significant difference (LSD) test. Categorical variables were compared between groups with the chisquare test. We compared the relationship between pain thresholds and headache frequency, as well as the differences in pain thresholds and cyclic changes of pain thresholds between groups. All tests were two-tailed, and the significance level was set at $p<0.05$. The data were presented as means with standard deviations (SD) or standard errors (SE).

\section{Results}

\section{Participant demographics}

We recruited 104 migraine patients $(35.7 \pm 10.0$ years, F/M: 85/19) and $32 \mathrm{HCs}(37.8 \pm 7.0$ years, F/M: 27/5). Twelve migraine patients were excluded from analyses due to incomplete baseline headache diaries. The final sample for analysis included 92 migraine patients with a $97.2 \pm 6.4 \%$ baseline headache diaries completion rate (range from $78.6 \%$ to $100 \%$ ). There were $28 \mathrm{CM}$ patients $(37.3 \pm 11.4$ years, F/M: 24/4) and 64 EM patients $(34.2 \pm 8.7$ years, $\mathrm{F} / \mathrm{M}: 52 / 12)$. Table 1 shows the demographics and the clinical data of patients and HC groups. No significant differences were found between groups regarding age and sex. The headache frequency $(p<0.001)$, HADS_A $(p<0.001)$, and HADS_D $(p=0.001)$ were significantly higher in the $\mathrm{CM}$ group compared with the EM group.

\section{Pain thresholds}

Table 2 shows HPTs, CPTs, and MPPTs of all three groups. EM participants had higher MPPTs compared with $\mathrm{CM}$ participants in both dermatomes (V1: $115.8 \pm 41.0$ vs. $94.5 \pm 34.5, p=0.021$ and $\mathrm{T} 1$ : $127.0 \pm 72.5$ vs. $85.5 \pm 33.8, p=0.003)$ and HCs in V1 dermatome (115.8 \pm 41.0 vs. $96.0 \pm 43.9, p=0.025)$. Pain thresholds were similar between CM participants and HCs. Of note, the results of MPPT were similar after logarithmic transformation, and for clearer

Table I. Demographics and clinical profiles of study subjects.

\begin{tabular}{|c|c|c|c|c|}
\hline & $\begin{array}{l}\text { All migraine } \\
\mathrm{n}=92\end{array}$ & $\begin{array}{l}C M \\
n=28\end{array}$ & $\begin{array}{l}E M \\
n=64\end{array}$ & $\begin{array}{l}\mathrm{HC} \\
\mathrm{n}=32\end{array}$ \\
\hline Age, years & $35.1 \pm 9.6$ & $37.3 \pm 11.4$ & $34.2 \pm 8.7$ & $37.8 \pm 7.0$ \\
\hline Gender, F/M & $76 / 16$ & $24 / 4$ & $52 / 12$ & $27 / 5$ \\
\hline Headache frequency, days/month & $12.3 \pm 8.8$ & $24.3 \pm 4.1^{*}$ & $7.0 \pm 3.6 *$ & $\mathrm{~N} / \mathrm{A}$ \\
\hline HADS_A $A^{\dagger}$ & $7.1 \pm 3.9$ & $9.3 \pm 3.7^{\ddagger}$ & $6.1 \pm 3.6^{\ddagger}$ & $3.0 \pm 2.2^{\ddagger}$ \\
\hline HADS_D ${ }^{\dagger}$ & $4.8 \pm 3.2$ & $6.4 \pm 3.2^{\ddagger}$ & $4.0 \pm 2.9^{\ddagger}$ & $2.8 \pm 2.6$ \\
\hline MIDAS & $23.6 \pm 35.1$ & $37.6 \pm 56.1^{*}$ & $17.4 \pm 17.5^{*}$ & $\mathrm{~N} / \mathrm{A}$ \\
\hline
\end{tabular}

$* p<0.00$ I, CM vs. EM.

tone-way ANOVA, both $p<0.001$.

${ }_{\text {p}}$ post-hoc LSD, CM vs. EM, CM vs. HC, EM vs. HC, all $p<0.05$.

Note: Data presented as mean \pm SD, 'all migraine' stands for the pooled data of patients with both chronic and episodic migraine excluding those with incomplete headache diary.

CM: chronic migraine; EM: episodic migraine; HC: healthy control; N/A: not applicable; HADS_A: anxiety section of Hospital Anxiety and Depression Scale; HADS_D: depression section of Hospital Anxiety and Depression Scale; MIDAS: Migraine Disability Scale. 
Table 2. Pain thresholds compared between groups.

\begin{tabular}{ccccc}
\hline & $\begin{array}{l}\text { All migraine } \\
\mathrm{n}=92\end{array}$ & $\begin{array}{l}\mathrm{CM} \\
\mathrm{n}=28\end{array}$ & $\begin{array}{l}\mathrm{EM} \\
\mathrm{n}=64\end{array}$ & $\begin{array}{l}\mathrm{HC} \\
\mathrm{n}=32\end{array}$ \\
\hline $\mathrm{VI}$ & & & & \\
MPPT $(\mathrm{g})^{*}$ & $109.3 \pm 40.2$ & $94.5 \pm 34.5$ & $115.8 \pm 41.0^{\dagger \ddagger}$ & $96.0 \pm 43.9$ \\
HPT $\left({ }^{\circ} \mathrm{C}\right)$ & $40.8 \pm 3.8$ & $40.2 \pm 3.8$ & $41.2 \pm 3.7$ & $42.2 \pm 4.2$ \\
CPT $\left({ }^{\circ} \mathrm{C}\right)$ & $21.4 \pm 7.6$ & $22.8 \pm 7.3$ & $20.4 \pm 7.8$ & $22.5 \pm 14.6$ \\
TI & & & & $103.7 \pm 54.9$ \\
MPPT $(\mathrm{g})^{*}$ & $114.4 \pm 65.9$ & $85.5 \pm 33.8$ & $127.0 \pm 72.5^{\dagger \neq}$ & $41.1 \pm 3.7$ \\
HPT $\left({ }^{\circ} \mathrm{C}\right)$ & $40.4 \pm 4.0$ & $39.8 \pm 3.7$ & $40.8 \pm 4.2$ & $19.2 \pm 10.2$ \\
CPT $\left({ }^{\circ} \mathrm{C}\right)$ & $20.0 \pm 8.4$ & $21.3 \pm 8.3$ & $19.0 \pm 8.6$ & \\
\hline
\end{tabular}

Note: Data presented as mean $\pm S D$, 'all migraine' stands for the pooled data of CM and EM.

*One-way ANOVA, $p<0.05$.

${ }^{\dagger}$ Post-hoc LSD, CM vs. EM, $p<0.05$.

‡Post-hoc LSD, EM vs. HC, $p<0.05$.

CM: chronic migraine; EM: episodic migraine; HC: healthy control; VI: first division of the 5th cranial nerve dermatome; TI: Ist thoracic nerve dermatome; MPPT: mechanical punctate pain threshold; HPT: heat pain threshold; CPT: cold pain threshold.

comparisons we presented our results with raw values. There were no significant differences between groups regarding HPT or CPT.

\section{Relationships between MPPTs and headache frequency}

The MPPTs decreased as headache frequency increased and nadired (Figure 1). In the EM group, headache frequency was negatively correlated with MPPTs in both $\mathrm{V} 1$ and $\mathrm{T} 1$ dermatomes $(\mathrm{r}=-0.457, p<0.001$ and $\mathrm{r}=-0.518, p<0.001)$. On the other hand, there was no significant correlation between headache frequency and MPPTs in the CM group.

\section{Migraine phases and MPPTs}

Among all 28 patients with CM, only one of them was in the preictal phase and the rest were in the ictal phase. Comparison between phases was not possible due to the imbalance of distribution; therefore, comparisons among migraine phases were only performed in EM participants. Among patients with EM, 16 were in the interictal phase, 20 in the preictal phase, 24 in the ictal phase, and four in the postictal phase. MPPTs varied by migraine phase in V1 $(p<0.001)$ and $\mathrm{T} 1(p<0.001)$ dermatomes. MPPTs in both dermatomes were higher during the interictal phase compared with the preictal $(p=0.021$ and 0.003 for $\mathrm{V} 1$ and T1), ictal $(p<0.001$ and $<0.001$ for $\mathrm{V} 1$ and T1), and postictal $(p<0.001$ and $<0.001$ for V1 and T1) phases (Table 3 ). In both V1 $(p=0.001)$ and T1 $(p<0.001)$ dermatomes, MPPTs were significantly higher in EM patients during the interictal phase compared with HCs. Pain thresholds in the preictal, ictal, or postictal phases of EM patients were similar compared to HCs.

\section{Contributions of migraine phases and headache frequency to MPPT}

Predictors of MPPTs were determined by stepwise linear analyses. Migraine phase was the strongest predictor of MPPTs in both V1 and T1 dermatomes $(\beta=-0.369, p=0.003$ and $\beta=-0.416, p<0.001)$, followed by headache frequency $(\beta=-0.298, p=0.014$ and $\beta=-0.338, p=0.004)$. The two-variable models showed that migraine phase and headache frequency independently explained $29.7 \%$ and $38.9 \%$ of the variance in MPPTs in V1 and T1 dermatomes, respectively.

\section{Discussion}

This study sought to investigate how headache frequency and migraine phase are related to HPT, CPT, and MPPT in a heterogeneous population of migraine patients. We found that a) QST results did not differ between $\mathrm{HCs}$ and patients with $\mathrm{CM}$; b) MPPTs decreased with increasing headache frequency in patients with EM; c) MPPT differed in different migraine phases and decreased when approaching the ictal phase in patients with EM. Migraine phases and headache frequency together accounted for $30-40 \%$ of total MPPT variance in EM patients. However, no similar results were found for heat- or cold-induced pain thresholds in our migraine patients.

Previous studies evaluating the relationship between headache frequency and pain sensitivity are limited, and studies that do assess these relationships are often inconsistent. For example, HPT and headache frequency appear to be unrelated in a study conducted by Schwedt and colleagues (9); however, patients with high headache frequency are more sensitive to heat 


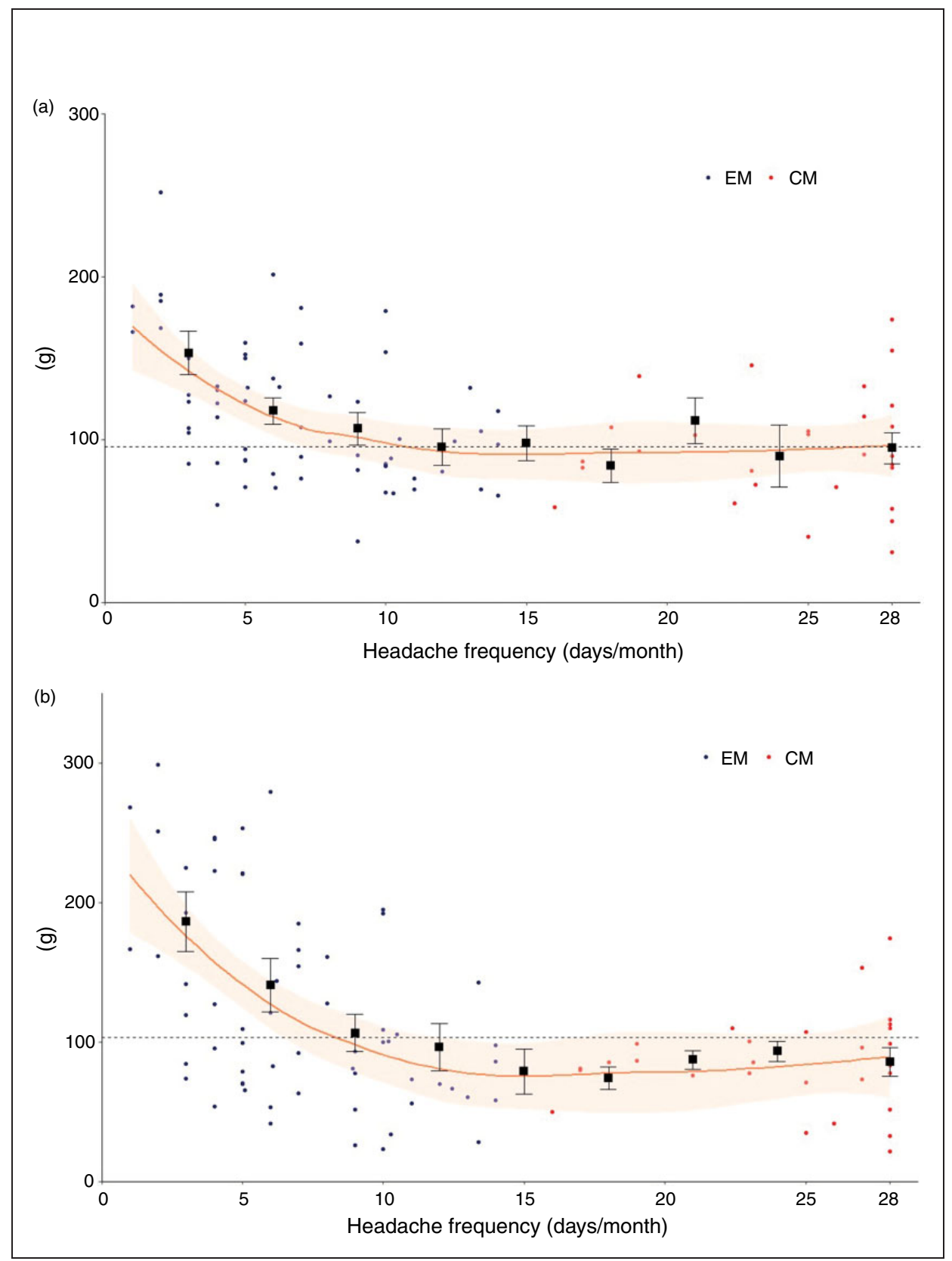

Figure I. The relationship between headache frequency and the mechanical punctate pain thresholds (MPPTs) of (a) VI and (b) TI dermatomes. (a) and (b) show the pooled data of all migraine patients. The broken black lines represent the MPPT of healthy controls. The black rectangular and the solid lines represent the mean and standard error of MPPTs within I-3, 4-6, .., 22-24, and $>24$ monthly headache days.

EM: episodic migraine; CM: chronic migraine.

pain in Mathur and colleagues' study (20). The current study found that MPPT's relationship with headache frequency differed depended on headache frequency. Specifically, as headache frequency increased, MPPT declined gradually and reached nadir. More studies with larger sample sizes are warranted to help us elucidate the relationship between pain sensitivities and headache frequency.
Migraine patients exhibited MPPTs during the interictal phase in both $\mathrm{V} 1$ and $\mathrm{T} 1$ dermatomes that were significantly higher than any other phases. However, the MPPTs declined at the preictal phase and remained low during ictal and postictal phases. The MPPTs in migraine patients were compatible with the mean values of HCs during peri-ictal and ictal phases. However, the number of patients in the ictal and 
Table 3. MPPT during different phases in patients with episodic migraine.

\begin{tabular}{lllll}
\hline & Interictal & Preictal & Ictal & Postictal \\
& $\mathrm{n}=16$ & $\mathrm{n}=20$ & $\mathrm{n}=24$ & $\mathrm{n}=4$ \\
\hline $\mathrm{VI} *$ & $145.9 \pm 52.4^{\dagger}$ & $117.0 \pm 23.9^{\ddagger}$ & $102.2 \pm 34.0$ & $70.7 \pm 10.7$ \\
TI* & $191.5 \pm 71.6^{\dagger}$ & $127.3 \pm 63.7$ & $94.7 \pm 53.9$ & $61.5 \pm 22.3$ \\
\hline
\end{tabular}

Note: Data presented as mean $\pm \mathrm{SD}$.

*One-way ANOVA, both $p<0.001$.

${ }^{\dagger}$ Post-hoc LSD, interictal vs. preictal, ictal, and postictal, VI $p=0.02 \mathrm{I},<0.00 \mathrm{I},<0.00 \mathrm{I}$ and $\mathrm{TI} p=0.003,<0.00 \mathrm{I},<0.00 \mathrm{I}$.

†Post-hoc LSD, preictal vs. postictal, $p=0.023$.

MPPT: mechanical punctate pain threshold; VI: first division of the 5 th cranial nerve dermatome; TI: Ist thoracic nerve dermatome.

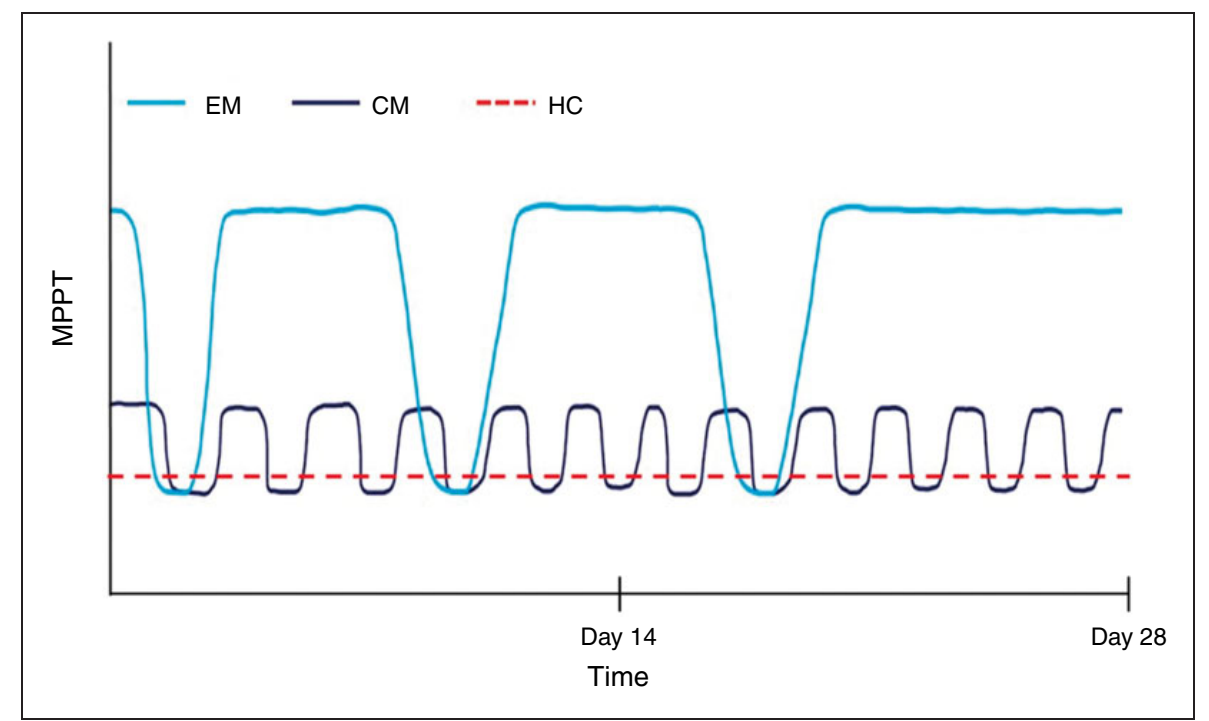

Figure 2. A concept map illustrates the mechanical punctate pain thresholds (MPPT) change in migraine patients with different headache frequencies.

EM: episodic migraine; CM: chronic migraine; HC: healthy control.

postictal phases are relatively few. In contrast, to define the phases of CM patients for the phase-wise analysis is difficult, because many of them barely possessed an interictal phase (Figure 2). Therefore, many patients with $\mathrm{CM}$ are at a stage of never-ending migraine attacks around peri-ictal phases; that is, from preictal to postictal stages $(26,27)$.

The current study has some methodological differences compared with previous MPPT studies. For example, many previous studies use von Frey hairs $(17,23,28,29)$, very few used electronic von Frey devices $(24,30)$. The MPPT of our HCs (103.7 g) was comparable with previous study (107.9 g) (30) using the same rigid tip with similar electronic device tested on the forearm. We did not compare the values from the study of Reitz and colleagues (24) because their testing area was the foot, which was not included in our study. On the other hand, in the current study, HC HPTs were similar to the previously reported values (face: $42.2 \pm 4.2^{\circ} \mathrm{C}$ vs. $41.5 \pm 4.1^{\circ} \mathrm{C}$; hand: $41.1 \pm$ $3.7^{\circ} \mathrm{C}$ vs. $\left.42.6 \pm 3.3^{\circ} \mathrm{C}\right)(23)$.

HPT and CPT appeared to have no consistent relationship with clinical profiles among patients with migraine in the current study, similar to previous studies (17-19). We do not know the exact mechanisms accounting for the discrepancy of clinical correlations for migraine patients in comparison with MPPT in our study. Various receptors and fibers are responsible for different types of pain (31): heat pain signals are largely transferred along $\mathrm{C}$ fibers, whereas mechanical pain and cold pain signals are mainly transferred along A delta fibers. Even with the same transfer fibers, transient receptor potential cation channel subfamily $M$ (melastatin) member 8 serves as the transduction channel of cold-induced pain (32), whereas other receptors such as potassium channel subfamily K member 4 (33), transient receptor potential cation channel subfamily $\mathrm{V}$ member 4 (34), and so on, are responsible for 
mechanical punctate pain. These differences may lead to the inconsistency of pain threshold changes in patients with migraine. However, further studies are needed to confirm these hypotheses.

We provide a concept figure of our study results (Figure 2) in order to link with potential clinical practice. By conducting longitudinal MPPT assessments on patients with EM, we may be able to predict a patient's next migraine attack. In addition, if a patient with EM starts to show constant decreasing MPPT, he or she may be in the early stages of chronification. With this in mind, clinicians could use some of these data to initiate early detection and prevention of chronic migraine. More importantly, our study demonstrated that mechanical pain sensitivities differed with different headache profiles, which likely explains, in part, why previous studies do not show consistent results.

Our study does, however, have limitations. First, our data were collected cross-sectionally, which may result in less robust relationships between pain thresholds and headache phases. A longitudinal study design should be considered for future research. Second, the number of patients in the postictal phase was small compared with the other phases. This was inevitable as our study was cross-sectional and pre-scheduled, but can be averted with longitudinal study design. Finally, because the current study was a single-center hospital-based study, generalizing of our study results should be cautious.

\section{Conclusion}

Collectively, our data suggest that MPPT is determined by headache frequency and migraine phases, and MPPTs decline when migraine patients approach ictal stage or their headache become frequent. However, future research, including a longitudinal study, is warranted to confirm our study findings.

\section{Clinical implications}

- MPPT is determined by headache frequency and migraine phases.

- Our study demonstrated that mechanical pain sensitivities differed with different headache profiles, which likely explains, in part, why previous studies do not show consistent results.

- We may be able to predict the next headache attack or chronification of migraine in patients by assessing the MPPT. Future longitudinal studies are warranted to verify the predictability.

\section{Acknowledgement}

We wish to thank all individuals in this study for their generous participation.

\section{Declaration of conflicting interests}

The authors declared no potential conflicts of interest with respect to the research, authorship, and/or publication of this article.

\section{Funding}

The authors disclosed receipt of the following financial support for the research, authorship, and/or publication of this article: Ministry of Science and Technology of Taiwan [MOST 108-2321-B-010 -014 -MY2 and 108-2321-B-010 001 and 108-2314-B-010-023-MY3] (to SJW) \& [MOST 107-2314B-010-021 and 108-2314-B-010-022-MY3] (to SPC); Ministry of Health and Welfare, Taiwan [MOHW 106-TDU-B-211113001, MOHW 107-TDU-B-211-123001, MOHW 108TDU-B-211-133001, MOHW 109-TDU-B-211-114001] (to KLL); Taipei Veterans General Hospital, Taiwan [VGH 108-C-092 and VGH 109-C-096] (to YFW); this work was supported by the Brain Research Center, National YangMing University from The Featured Areas Research Center Program within the framework of the Higher Education
Sprout Project by the Ministry of Education (MOE) in Taiwan. The funders had no role in study design, data collection and analysis, decision to publish, or preparation of the manuscript.

\section{ORCID iDs}

Li-Ling Hope Pan (D) https://orcid.org/0000-0002-0192-389X Kuan-Lin Lai (D) https://orcid.org/0000-0002-2436-6555 Shih-Pin Chen (D) https://orcid.org/0000-0003-3492-9902 Yu-Hsiang Ling (D) https://orcid.org/0000-0003-2360-0693 Shuu-Jiun Wang (D) https://orcid.org/0000-0001-5179-5358

\section{References}

1. GBD 2016 Disease and Injury Incidence and Prevalence Collaborators. Global, regional, and national incidence, prevalence, and years lived with disability for 328 diseases and injuries for 195 countries, 1990-2016: A systematic analysis for the Global Burden of Disease Study 2016. Lancet 2017; 390: 1211-1259.

2. Cao Z, Lai K-L, Lin C-T, et al. Exploring resting-state EEG complexity before migraine attacks. Cephalalgia 2018; 38: 1296-1306.

3. Brighina F, Palermo A and Fierro B. Cortical inhibition and habituation to evoked potentials: relevance for pathophysiology of migraine. J Headache Pain 2009; 10: 77-84. 
4. Borsook D, Veggeberg R, Erpelding N, et al. The insula: A "hub of activity" in migraine. Neuroscientist 2016; 22: 632-652.

5. de Tommaso M, Ambrosini A, Brighina F, et al. Altered processing of sensory stimuli in patients with migraine. Nat Rev Neurol 2014; 10: 144-155.

6. Nahman-Averbuch H, Shefi T, Schneider VJ, et al. Quantitative sensory testing in patients with migraine: A systematic review and meta-analysis. Pain 2018; 159: 1202-1223.

7. Peng K-P and May A. Migraine understood as a sensory threshold disease. Pain 2019; 160: 1494-1501.

8. Schwedt TJ, Krauss MJ, Frey K, et al. Episodic and chronic migraineurs are hypersensitive to thermal stimuli between migraine attacks. Cephalalgia 2011; 31: 6-12.

9. Schwedt TJ, Zuniga L and Chong CD. Low heat pain thresholds in migraineurs between attacks. Cephalalgia 2015; 35: 593-599.

10. Uthaikhup S, Sterling M and Jull G. Widespread sensory hypersensitivity is not a feature of chronic headache in elders. Clin J Pain 2009; 25: 699-704.

11. Chaves TC, Dach F, Florencio LL, et al. Concomitant migraine and temporomandibular disorders are associated with higher heat pain hyperalgesia and cephalic cutaneous allodynia. Clin J Pain 2016; 32: 882-888.

12. Engstrøm M, Hagen K, Bjørk MH, et al. Sleep quality, arousal and pain thresholds in migraineurs: A blinded controlled polysomnographic study. J Headache Pain 2013; 14: 12 .

13. Uglem M, Omland PM, Nilsen KB, et al. Does pain sensitivity change by migraine phase? A blinded longitudinal study. Cephalalgia 2017; 37: 1337-1349.

14. Barón J, Ruiz M, Palacios-Ceña M, et al. Differences in topographical pressure pain sensitivity maps of the scalp between patients with migraine and healthy controls. Headache 2017; 57: 226-235.

15. Fernández-de-las-Peñas $\mathrm{C}$, Arendt-Nielsen L, Cuadrado ML, et al. Generalized mechanical pain sensitivity over nerve tissues in patients with strictly unilateral migraine. Clin J Pain 2009; 25: 401-406.

16. Schwedt TJ and Chong CD. Correlations between brain cortical thickness and cutaneous pain thresholds are atypical in adults with migraine. PloS One 2014; 9: e99791.

17. Weissman-Fogel I, Sprecher E, Granovsky Y, et al. Repeated noxious stimulation of the skin enhances cutaneous pain perception of migraine patients in-between attacks: Clinical evidence for continuous sub-threshold increase in membrane excitability of central trigeminovascular neurons. Pain 2003; 104: 693-700.

18. Sand T, Zhitniy N, Nilsen KB, et al. Thermal pain thresholds are decreased in the migraine preattack phase. Eur J Neurol 2008; 15: 1199-1205.
19. Beese LC, Putzer D, Osada N, et al. Contact heat evoked potentials and habituation measured interictally in migraineurs. J Headache Pain 2015; 16: 1.

20. Mathur VA, Moayedi M, Keaser ML, et al. High frequency migraine is associated with lower acute pain sensitivity and abnormal insula activity related to migraine pain intensity, attack frequency, and pain catastrophizing. Front Hum Neurosci 2016; 10: 489.

21. Headache Classification Committee of the International Headache Society (IHS). The International Classification of Headache Disorders, 3rd edition (beta version). Cephalalgia 2013; 33: 629-808.

22. The International Classification of Headache Disorders, 3rd edition. Cephalalgia 2018; 38: 1-211.

23. Rolke R, Baron R, Maier C, et al. Quantitative sensory testing in the German Research Network on Neuropathic Pain (DFNS): Standardized protocol and reference values. Pain 2006; 123: 231-243.

24. Reitz M-C, Hrncic D, Treede R-D, et al. A comparative behavioural study of mechanical hypersensitivity in 2 pain models in rats and humans. Pain 2016; 157: 12481258.

25. Tassorelli C, Diener H-C, Dodick DW, et al. Guidelines of the International Headache Society for controlled trials of preventive treatment of chronic migraine in adults. Cephalalgia 2018; 38: 815-832.

26. Schoenen J. Is chronic migraine a never-ending migraine attack? Pain 2011; 152: 239-240.

27. Chen W-T, Wang S-J, Fuh J-L, et al. Persistent ictal-like visual cortical excitability in chronic migraine. Pain 2011; 152: 254-258.

28. Burstein R, Yarnitsky D, Goor-Aryeh I, et al. An association between migraine and cutaneous allodynia. Ann Neurol 2000; 47: 614-624.

29. Cooke L, Eliasziw M, Becker WJ. Cutaneous allodynia in transformed migraine patients. Headache 2007; 47: 531-539.

30. Chassaing C, Schmidt J, Eschalier A, et al. Hyperalgesia induced by cutaneous freeze injury for testing analgesics in healthy volunteers. Br J Clin Pharmacol 2006; 61: 389-397.

31. Dubin AE and Patapoutian A. Nociceptors: The sensors of the pain pathway. J Clin Invest 2010; 120: 3760-3772.

32. Bautista DM, Siemens J, Glazer JM, et al. The menthol receptor TRPM8 is the principal detector of environmental cold. Nature 2007; 448: 204-208.

33. Maingret F, Fosset $M$, Lesage $F$, et al. TRAAK is a mammalian neuronal mechano-gated $\mathrm{K}+$ channel. J Biol Chem 1999; 274: 1381-1387.

34. Alessandri-Haber N, Joseph E, Dina OA, et al. TRPV4 mediates pain-related behavior induced by mild hypertonic stimuli in the presence of inflammatory mediator. Pain 2005; 118: 70-79. 